\title{
Hungry pigeons make suboptimal choices, less hungry pigeons do not
}

\author{
Jennifer R. Laude • Kristina F. Pattison • \\ Thomas R. Zentall
}

Published online: 26 June 2012

(C) Psychonomic Society, Inc. 2012

\begin{abstract}
Hungry animals will often choose suboptimally by being attracted to reliable signals for food that occur infrequently (they gamble) over less reliable signals for food that occur more often. That is, pigeons prefer an option that $50 \%$ of the time provides them with a reliable signal for the appearance of food but $50 \%$ of the time provides them with a reliable signal for the absence of food (overall $50 \%$ reinforcement) over an alternative that always provides them with a signal for the appearance of food $75 \%$ of the time (overall $75 \%$ reinforcement). The pigeons appear to choose impulsively for the possibility of obtaining the reliable signal for reinforcement. There is evidence that greater hunger is associated with greater impulsivity. We tested the hypothesis that if the pigeons were less hungry, they would be less impulsive and, thus, would choose more optimally (i.e., on the basis of the overall probability of reinforcement). We found that hungry pigeons choose the $50 \%$ reinforcement alternative suboptimally but less hungry pigeons prefer the more optimal $75 \%$ reinforcement. Paradoxically, pigeons that needed the food more received less of it. These findings have implications for how level of motivation may also affect human suboptimal choice (e.g., purchase of lottery tickets and playing slot machines).
\end{abstract}

Keywords Gambling · Choice behavior - Suboptimal choice $\cdot$ Signaled reinforcement $\cdot$ Pigeons

J. R. Laude $\cdot$ K. F. Pattison • T. R. Zentall

University of Kentucky,

Lexington, KY 40506-0044, USA

T. R. Zentall ( ()

Department of Psychology, University of Kentucky,

Lexington, KY 40506-0044, USA

e-mail: zentall@uky.edu
Maladaptive gambling by humans can be defined as making a decision to choose a low-probability but high-payoff alternative over a high-probability, low-payoff alternative, such that the net expected return is less than what one has wagered. In choosing the first alternative, odds are against the gambler (ratio of negative outcomes compared with positive outcomes) such that, in the long term, this decision will result in losing more than winning. Examples of this include when humans purchase a lottery ticket or gamble at a casino in which, on average, engaging in repeated gambles results in a loss of money. Research that we have conducted with pigeons appears to be analogous to human gambling behavior of this kind.

Gipson, Alessandri, Miller, and Zentall (2009) found that pigeons preferred a discriminative stimulus alternative when choice of that alternative resulted in a substantial loss of reinforcement. The choice was between $50 \%$ reinforcement with discriminative stimuli and $75 \%$ reinforcement with nondiscriminative stimuli. A reliable suboptimal preference for the $50 \%$ reinforcement alternative was found, in spite of the fact that the pigeons could have received $50 \%$ more food by choosing the nondiscriminative-stimulus alternative. More recently, Stagner and Zentall (2010) gave pigeons a choice between $20 \%$ reinforcement with discriminative stimuli and $50 \%$ reinforcement with nondiscriminative stimuli and found that pigeons preferred the discriminativestimulus alternative in spite of the fact that they could have earned 2.5 times as much food by choosing the $50 \%$ reinforcement alternative.

In the Gipson et al. (2009) and Stagner and Zentall (2010) experiments, the appearance of the discriminative stimuli indicated whether the pigeons would be fed or not, but choice of the nondiscriminative stimuli did not clarify whether food would follow. Thus, it may be that the 
preference for the low probability of reinforcement alternative actually resulted from avoidance of the ambiguous nondiscriminative alternative. To test this hypothesis, Zentall and Stagner (2011) manipulated the magnitude of reinforcement rather than the percentage of reinforcement. For the lower probability of reinforcement alternative with discriminative stimuli, on $20 \%$ of the trials, a stimulus was presented that always predicted 10 pellets of food, and on the remaining trials, a stimulus was presented that always predicted the absence of food. Thus, the mean reinforcement per trial associated with this alternative was 2 pellets of food. Choice of the other alternative produced a stimulus that always predicted 3 pellets of food. Thus, the choice was between an average of 2 pellets for the alternative with discriminative stimuli and a constant 3 pellets for the alternative with nondiscriminative stimuli. Although the alternative with nondiscriminative stimuli now predicted a certain outcome, a reliable suboptimal preference for the 2-pellet alternative was found.

To show that the pigeons' preference for the 2-pellet alternative with discriminative stimuli did not result from a preference for a variable schedule of reinforcement (sometimes 10 pellets, sometimes none) over a constant 3 pellets, Zentall and Stagner (2011) converted the discriminative-stimulus alternative into a nondiscriminative-stimulus alternative by following each of the formerly discriminative stimuli with 10 pellets, $20 \%$ of the time. After several sessions, the pigeons reversed their preference and reliably chose the alternative that provided a certain 3 pellets over the alternative that provided a variable outcome that averaged 2 pellets.

To determine whether the task developed for pigeons was appropriate for use with humans, we indentified a sample of undergraduates who had indicated that, either weekly or daily, they engaged in gambling-related activities and matched them with participants who reported that they never engaged in gambling-related activities. Using a paradigm very similar to that in Stagner and Zentall (2010), we found that self-described gamblers chose the suboptimal alternative significantly more than did self-described nongamblers (Molet et al., in press). Thus, the pigeon task appears to be a suitable analog of human gambling because, like pigeons, humans who gamble show a tendency to make suboptimal choices on this gambling-like task.

The findings that pigeons maintained on a moderately restricted diet and humans who report that they frequently engage in gambling-related activities sacrifice considerable opportunity for reinforcement to obtain discriminative stimuli if one of them predicts a high probability or large amount of reinforcement are inconsistent with the law of effect (Thorndike, 1933). That is, if effort and delay of reinforcement are held constant, animals should favor alternatives that provide higher probabilities (or greater amounts) of reinforcement.
One account of these results is that the conditioned reinforcement associated with the immediacy of the appearance of the stimulus that reliably predicts a high probability (or high magnitude) of reinforcement is sufficient to produce a preference for that alternative. Thus, in spite of the fact that the actual delay to reinforcement is equated for the two alternatives, the immediacy of the appearance of the conditioned reinforcer following choice may make the choice more analogous to a choice between a low-probability immediate reinforcer (i.e., the conditioned reinforcer) and a more probable delayed reinforcer (i.e., the actual primary reinforcement associated with the other alternative) (Rachlin, Logue, Gibbon, \& Frankel, 1986; Rachlin, Raineri, \& Cross, 1991). Consistent with this interpretation, Ainslie (1974) found that such a delay of primary reinforcement with pigeons often results in a steep temporal discounting function (the plot of how much greater the probably reinforcement at a delay must be to compensate for a lower probability immediate reinforcer). The steepness of the discounting function can be taken as a measure of the degree to which an animal is characterized as impulsive or, the degree to which it lacks self-control (Hull, 1943). That is to say, behavioral measures of discounting are often interpreted as indicating the degree of impulsivity. Given that our task appears to be a suitable analog of human gambling behavior, the finding that there is a relation between degree of impulsivity as measured by delay-discounting tasks and pathological gambling behavior (Alessi \& Petry, 2003; Petry, 2001; Petry \& Casarella, 1999) supports the hypothesis that impulsivity acts as a proximal mechanism, resulting in a preference for the less-optimal alternative.

Herrnstein (1981) proposed that the value of immediacy should increase with the level of motivation, and there is evidence that greater levels of food restriction are associated with greater rates of delay discounting (Eisenberger, Masterson, \& Lowman 1982). That is, hungry animals tend to show a greater preference for immediate rewards (see also Bradshaw \& Szabadi, 1992; Snyderman, 1983). However, other research suggests that level of motivation has little effect on the preference for small immediate reinforcers over larger delayed reinforcers (Logue, Chavarro, Rachlin, \& Reeder, 1988; Logue \& Peña-Correal, 1985).

If impulsiveness is reduced by a reduction in motivation, it may be that pigeons will choose more optimally if they are less motivated. Thus, the purpose of the present experiment was to test the hypothesis that in the choice procedure used by Gipson et al. (2009), pigeons that are maintained on a less restricted diet will attribute less value to the conditioned reinforcer. If less value is attributed to the conditioned reinforcer, the pigeons may base their choice more on the overall probability of reinforcement associated with their initial choice and, thereby, will choose more optimally. 


\section{Method}

Subjects

The subjects were 16 White Carneaux pigeons that were retired breeders (4-12 years old). The pigeons had served previously in a matching-to-sample experiment with hue stimuli. All pigeons were given free access to mixed grains (Purina Pro Grains) until their weights stabilized (weight fluctuation $\leq 20 \mathrm{~g}$ for 5 consecutive days). The 5-day average then became each pigeon's free-feeding weight. On the basis of these weights, two levels of food restriction were calculated: $75 \%$ of free-feeding weight and $90 \%$ of free-feeding weight. Eight of the pigeons, randomly assigned, were maintained at $90 \%$ (or above) of their free-feeding weight, and the 8 remaining pigeons were maintained at $75 \%$ of their free-feeding weight. All pigeons were weighed prior to each experimental session. Pigeons in the $75 \%$ food restriction condition were permitted to run in their experimental session only if they were at or below weight, and pigeons in the $90 \%$ food restriction condition were permitted to run only if they were at or above weight (a total of three sessions were skipped because pigeons failed to meet this criterion, all in the $75 \%$ food restriction condition). The pigeons were individually housed in wire cages, with free access to water and grit, in a colony room that was maintained on a 12:12-h light:dark cycle. The pigeons were cared for in accordance with University of Kentucky animal care guidelines.

\section{Apparatus}

The experiment was conducted in two BRS/LVE (Laurel, MD) sound-attenuating standard operant test chambers with inside measurements of $35 \mathrm{~cm}$ high, $30 \mathrm{~cm}$ long, and $35 \mathrm{~cm}$ across the response panels. The response panel in each chamber had a horizontal row of three response keys $25 \mathrm{~cm}$ above the floor. The rectangular keys $(2.5 \mathrm{~cm}$ high, $33.0 \mathrm{~cm}$ wide $)$ were separated from each other by $1.0 \mathrm{~cm}$, and behind each key was a 12-stimulus inline projector (Industrial Electronics Engineering, Van Nuys, CA). The left and right projectors projected red, yellow, blue, green, and white hues (Kodak Wratten Filter Nos. 26, 9, 38, 60) and no filter, respectively. In each chamber, the bottom of the center-mounted feeder was $9.5 \mathrm{~cm}$ from the floor. When the feeders were raised, they were illuminated by $28-\mathrm{V}, 0.04-\mathrm{A}$ lamps. Reinforcement consisted of $1.5 \mathrm{~s}$ of Purina Pro Grains. Exhaust fans mounted on the outside of the chambers masked extraneous noise. A microcomputer in an adjacent room controlled the experiment. Half of the pigeons in each food restriction condition were trained in each chamber.
Pretraining

The pigeons were trained to peck each of the stimuli (red, yellow, blue, green, and white) on a fixed interval schedule (the first response after the specified time was reinforced) beginning at $1 \mathrm{~s}$ and increasing to 5 and $10 \mathrm{~s}$. During each of 10 pretraining sessions, the pigeons received 10 presentations of each stimulus. Trials were separated by a 10 -s darkened intertrial interval (ITI).

\section{Training}

On forced trials (24 trials per session), a white hue was presented on either the right or the left key. The other side key remained dark and was inoperative. One peck to the lit side key initiated the terminal link. For half of the pigeons in each group, if the white key on the left was illuminated, on half of the trials, one peck turned the white key red and, after $10 \mathrm{~s}$ (a response-independent, fixed-duration 10-s schedule), was followed by reinforcement. On the remaining left white-key trials, one peck turned the white key green for a fixed duration $(10 \mathrm{~s})$, followed by no reinforcement. If the white key on the right was illuminated, one peck turned the white key blue or yellow and, after $10 \mathrm{~s}$, was followed by reinforcement, $75 \%$ of the time (each hue occurred with a probability of .5). For the remaining pigeons in each group, the sides associated with the contingencies were reversed (see the design in Fig. 1). Randomly intermixed among the forced trials were 24 choice trials. During these trials, the left and right side keys were both illuminated white. Choice of one of the keys turned off the other key and rendered the unchosen key inoperative while the contingencies associated with the chosen key were in operation. On both forced and choice trials the initial-link stimuli were not terminated until the pigeons made a response. Forced and choice trials were separated by a 10 -s darkened ITI that included reinforcement when available. All pigeons were given 20 sessions of training with this task.

\section{Results}

The pigeons quickly developed a preference for one of the alternatives. By session 6, pigeons in the high-foodrestriction group showed a clear preference for the discriminative-stimulus alternative with $50 \%$ reinforcement, whereas pigeons in the low-food-restriction group showed a continuous preference for the nondiscriminativestimulus alternative with $75 \%$ reinforcement (see Fig. 2). For the pigeons in the high-food-restriction group, the preference for the discriminative-stimulus alternative was statistically reliable as of session $9, t(7)=3.02, p=.019$. For the pigeons in the low-food-restriction group, the preference for 


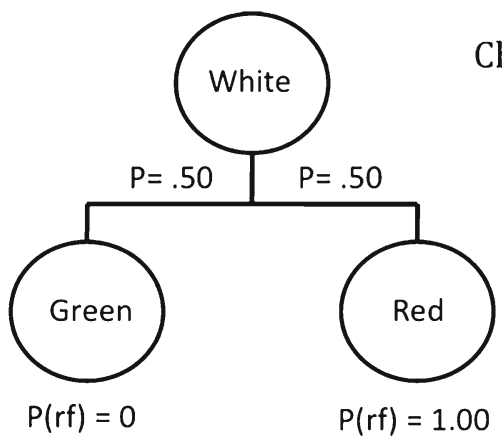

Fig. 1 Experimental design: Pigeons were given a choice between two alternatives. Choice of one alternative either produced a stimulus (e.g., red) that yielded $100 \%$ reinforcement on half of the trials or presented another stimulus (e.g., green) that was followed by the absence of reinforcement on the remaining half of the trials. Choice of the other

the nondiscriminative-stimulus alternative appeared on session 2 , and the preference was statistically reliable on session $3, t(7)=2.514, p=.04$. The difference between the two groups was statistically reliable by session $5, t(14)=2.4, p=.03$. When the data were pooled over the 20 sessions of training, there was a significant difference in choice behavior between pigeons maintained at a high level of food restriction, as compared with pigeons maintained at a low level of restriction, $t(14)=3.58, p=.003$.

Although, with continued experience with this task, the high-food-restriction group's preference for the discriminative-stimulus alternative appears to have gotten even stronger, for the low-food-restriction group, the preference for the nondiscriminative-stimulus alternative

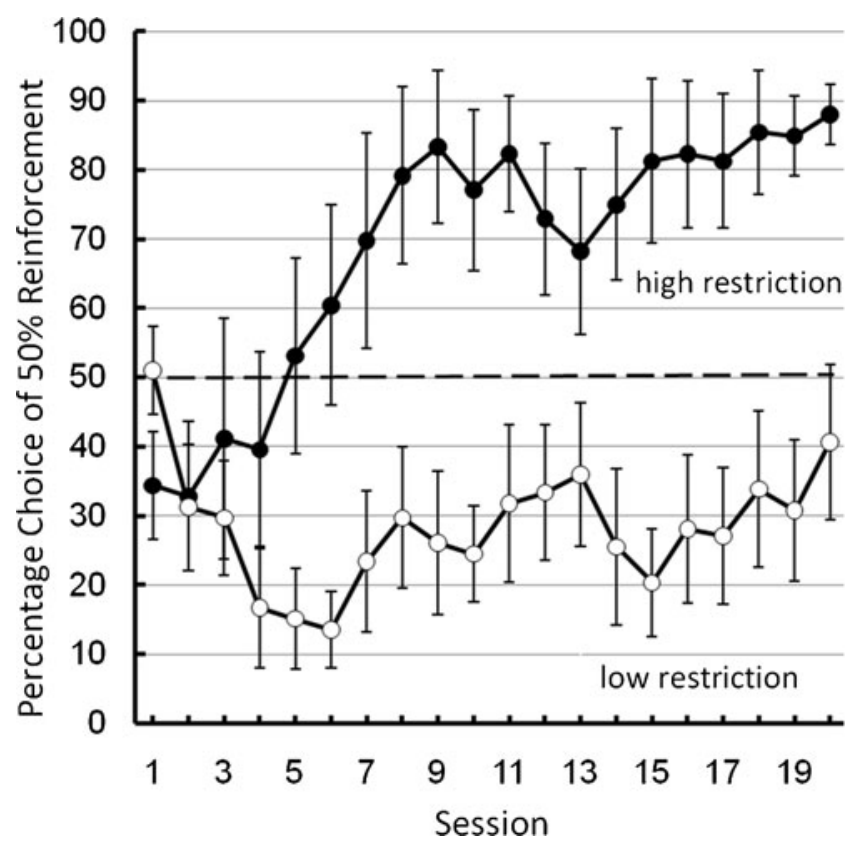

Fig. 2 Acquisition of the preference for the discriminative stimulus alternative for pigeons in the high- and low-food-restriction groups (error bars $= \pm 1$ standard error of the mean)

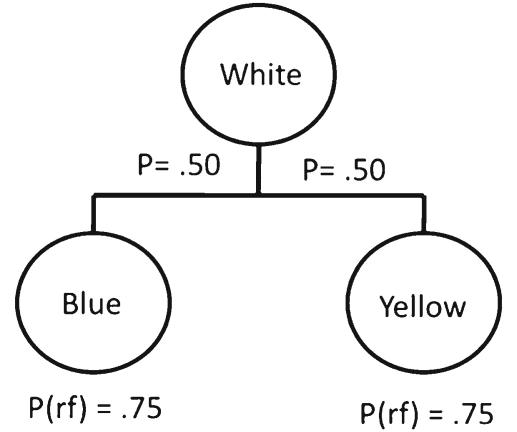

alternative was followed by one of two stimuli (e.g., blue or yellow), each of which yielded reinforcement $75 \%$ of the time. Both spatial location and colors were counterbalanced over pigeons in each food restriction group

appears to have gotten weaker. Whereas, for the low-fooddeprivation group, the preference for the nondiscriminative stimuli was highest on session 6 (86.5\%), it was relatively weak on session $20(59.4 \%)$. Thus, it may be that with continued training, the low-food-restriction pigeons would eventually show a preference for the suboptimal alternative as well. However, when the data were pooled over the last five sessions of training, the difference in preference between the two groups was still reliably different, $t(14)=4.26, p<.001$.

An indirect measure of the degree to which pigeons prefer one alternative over the other is the latency of response to the initial-link white stimulus on forced trials. Of course, regardless of the nature of the forced trial, latency to respond is likely to be strongly affected by level of food restriction. Thus, to obtain a relative latency score with which it would be appropriate to compare the two groups, we used the ratio of mean latency for the $75 \%$ reinforcement alternative to that for the $50 \%$ reinforcement alternative. Thus, if latency to respond was a reflection of choice preference, one would expect a preference for the suboptimal alternative to appear as a ratio greater than 1.0 , whereas a preference for the more optimal alternative would appear as a ratio less than 1.0. The mean ratio of latency to respond on forced trial, pooled over sessions 16-20, for the highfood-restriction pigeons was $2.72(S D=2.15)$ and for the low-food-restriction pigeons was $0.84(S D=0.84)$. An independent samples $t$-test revealed that the difference in ratio between the two groups was statistically significantly, $t$ $(14)=2.82, p=.01$. A single-sample $t$-test indicated that the mean ratio of latency to respond to the $75 \%$ alternative over the latency to respond to the $50 \%$ alternative was significantly greater than 1.0 for the high-restriction group, $t(7)=2.66, p=.03$, but was not significantly lower than 1.0 for the low-restriction group, $t<1$. The failure of the latency ratio for the low-restriction group to be significantly less than 1.0 is likely due to the fact that the preference for the optimal alternative for that group 
Table 1 Latencies to forced choice of $75 \%$ reinforcement alternative to forced choice of $50 \%$ reinforcement alternative, the ratio, and percentage choice of the low probability of reinforcement alternative for individual pigeons for sessions $16-20$

\begin{tabular}{|c|c|c|c|c|}
\hline \multirow[b]{2}{*}{ Bird No. } & \multicolumn{4}{|c|}{ Low Food Restriction } \\
\hline & $\begin{array}{l}\text { Latency to } \\
75 \% \text { alt. }\end{array}$ & $\begin{array}{l}\text { Latency } \\
50 \% \text { alt. }\end{array}$ & $\begin{array}{l}\text { Latency to } 75 \% \text { alt/ } \\
\text { Latency } 50 \% \text { alt. }\end{array}$ & $\begin{array}{l}\text { Percentage Choice } \\
\text { Low prob. alt. }\end{array}$ \\
\hline 17878 & 13.28 & 16.26 & 0.82 & 18.3 \\
\hline 20699 & 8.98 & 71.32 & 0.13 & 20.8 \\
\hline 583 & 209.23 & 176.59 & 1.18 & 35.8 \\
\hline 19229 & 86.81 & 128.45 & 0.68 & 94.2 \\
\hline 11746 & 11.57 & 16.78 & 0.69 & 28.3 \\
\hline 10534 & 3.08 & 2.37 & 1.30 & 31.7 \\
\hline 1382 & 35.09 & 22.02 & 1.59 & 0 \\
\hline 19836 & 5.71 & 19.87 & 0.29 & 27.5 \\
\hline \multirow[t]{2}{*}{ Mean } & 46.72 & 56.71 & 0.84 & 32.1 \\
\hline & \multicolumn{4}{|c|}{ High Food Restriction } \\
\hline Bird No. & $\begin{array}{l}\text { Latency to } \\
75 \% \text { alt. }\end{array}$ & $\begin{array}{l}\text { Latency } \\
50 \% \text { alt. }\end{array}$ & $\begin{array}{l}\text { Latency to } 75 \% \text { alt/ } \\
\text { Latency } 50 \% \text { alt. }\end{array}$ & $\begin{array}{l}\text { Percentage Choice } \\
\text { Low prob. alt. }\end{array}$ \\
\hline 15911 & 27.75 & 5.38 & 5.16 & 91.7 \\
\hline 581 & 3.16 & 2.04 & 1.55 & 90.0 \\
\hline 4217 & 10.54 & 1.78 & 5.92 & 100.0 \\
\hline 8993 & 7.24 & 7.31 & 0.99 & 35.8 \\
\hline 19205 & 1.69 & 0.99 & 1.71 & 79.2 \\
\hline 22642 & 1.16 & 0.76 & 1.53 & 79.2 \\
\hline 19243 & 2.02 & 0.76 & 2.66 & 100.0 \\
\hline 007 & 3.20 & 1.45 & 2.21 & 99.2 \\
\hline Mean & 7.10 & 2.56 & 2.72 & 84.4 \\
\hline
\end{tabular}

was not as strong as the preference for the suboptimal alternative for the high-food-restriction group.

If the ratio of latency to the forced $75 \%$ reinforcement alternative to the latency to the forced $50 \%$ reinforcement alternative reflects the relative preference for the two alternatives, one might expect there to be positive correlation between that ratio and the preference for the $50 \%$ reinforcement alternative. In fact, a statistically significant correlation was found (see Table 1), $r(14)=.69, p=.003$. Thus, when the pigeons are forced to choose their less preferred alternative, their latency to respond tends to be longer.

If the pigeons are actually choosing suboptimally, pigeons in the low-food-restriction group should be receiving more food than those in the high-food-restriction group. Given that half of the trials in each session were forced trials (24) and the difference in the number of possible reinforcements on choice trials was relatively small (18 reinforcements for $100 \%$ optimal choices vs. 12 reinforcements for $100 \%$ suboptimal choices), one can ask whether the difference in the number of reinforcements obtained by the pigeons in the two groups was statistically reliable. Although the difference was relatively small, when pooled over the 20 training sessions, the difference in the number of reinforcements per session between the low-restriction group
(34.3) and the high-restriction group (31.9) was statistically significant, $t(14)=3.57, p=.003$ (see Fig. 3).

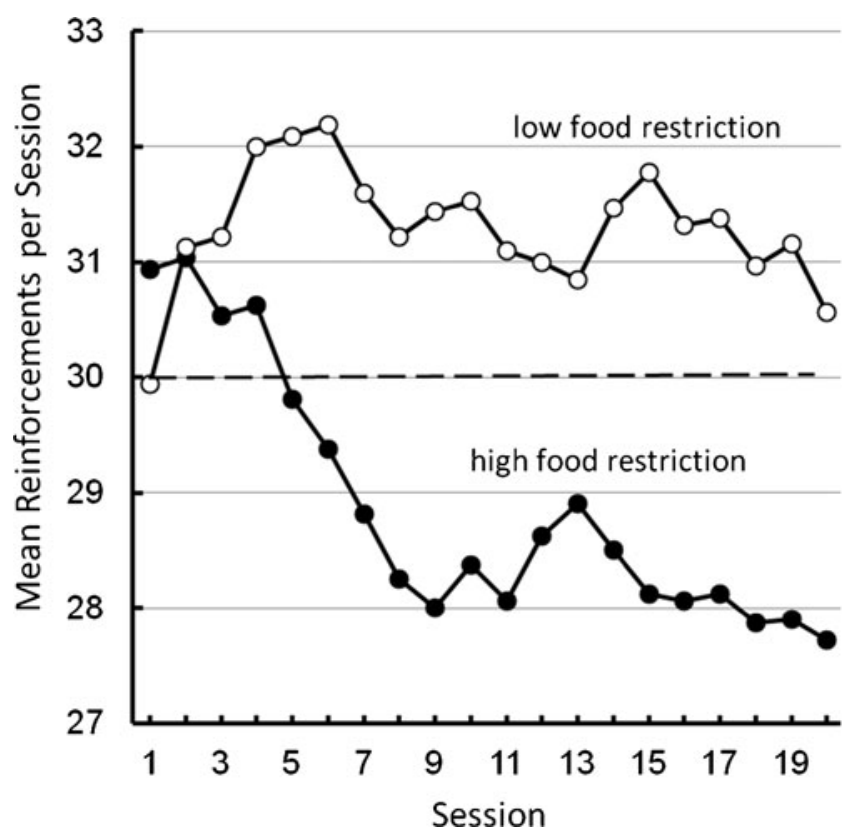

Fig. 3 Mean number of reinforcements per session for pigeons in the high- and low-food-restriction groups 


\section{Discussion}

The purpose of the present experiment was to test the hypothesis that level of motivation would affect the preference for the suboptimal discriminative-stimulus alternative over the more optimal nondiscriminative-stimulus alternative. The hypothesis was based on the assumption that the value of the discriminative-stimulus alternative was dependent on the immediacy of the conditioned reinforcer that predicted $100 \%$ reinforcement and, if the pigeons were less motivated, the conditioned reinforcer would have less value and the pigeons would be better able to judge the two alternatives in terms of the end of trial outcomes. To assess this possibility, we maintained pigeons at two levels of food restriction and then trained them on a version of the Gipson et al. (2009) procedure. The results confirmed that pigeons in the high-food-restriction group acquired a strong preference for the alternative that provided discriminative stimuli but only $50 \%$ reinforcement over the alternative that provided nondiscriminative stimuli but $75 \%$ reinforcement. However, pigeons in the low-food-restriction group acquired a strong preference for the alternative with nondiscriminative stimuli but a greater overall probability of reinforcement (75\%). Thus, it appears that pigeons in the high-food-restriction group based their choice on the immediacy of the appearance of the stimulus associated with a high probability of reinforcement, whereas pigeons in the low-food-restriction group based their choice on the overall probability of reinforcement associated with each alternative. In support of this interpretation of the results, Belke and Spetch (1994) reported that suboptimal choice by normally restricted pigeons was eliminated when a 5-s delay was imposed between the initial and terminal link stimuli.

Given appropriate experience, animals should become sensitive to the relative amounts of food obtained from different alternatives and choose optimally (optimal foraging theory; Stephens \& Krebs, 1986). This theory states that animals will forage in such a way as to maximize their net energy input by capturing and consuming food containing the most calories, while expending the least amount of energy in doing so. However, this theory does not consider how motivation affects choice. The present results could be interpreted in terms of models of risk-sensitive foraging. Specifically, it has been suggested that if a bird is on a negative energy budget (the rate of gain is not sufficiently high for the animal to survive with a fixed option), the animal's only chance of survival may be for it to be risk prone and gamble on the variable option (Stephens, 1981). But as Kacelnik and Bateson (1996) suggested, for pigeons trained under the present high-restriction conditions in which the majority of the daily ration is generally received in the experiment, the rate of food intake experienced is likely to be sufficient to result in a positive energy budget.
Kacelnik and Bateson proposed that predictions based on a negative energy budget would apply primarily to small birds with a high rate of metabolism that would likely die over night unless they were risk prone, rather than to larger birds, such as pigeons, that can go several days without food. Stephens's model indicates that if a bird is on a positive energy budget, it should be risk averse and thus, at the time of choice, it should prefer the alternative with the higher probability of reinforcement. In fact, we have found that when discriminative stimuli do not differentially signal reinforcement and its absence, pigeons do choose optimally (Stagner \& Zentall, 2010).

In the present procedure, assessment of the overall probability of reinforcement associated with the two alternatives requires that the pigeon accumulate the probability of reinforcement associated with each of the alternatives over trials, and that may be especially difficult for pigeons in the high-food-restriction group because they appear to have a shorter "time horizon" (Krebs \& Kacelnik, 1984) than do pigeons in the low-food-restriction group. This impaired ability to calculate odds is also characteristic of pathological gamblers and is likely due to a high degree of impulsivity. Support for the idea that increased impulsivity functions to increase maladaptive choice in this task can be seen in the mean ratios of latency to respond to the $75 \%$ alternative over latency to respond to the $50 \%$ alternative on forced trials for the two groups. More specifically, pigeons maintained on a high-restriction diet had significantly larger ratios (took longer to respond to the $75 \%$ reinforcement alternative on forced trials than to the $50 \%$ reinforcement alternative) than did pigeons maintained on a mildly restricted diet. These results suggest that pigeons maintained at high restriction responded more impulsively to the alternative associated with the immediacy of the conditioned reinforcer over the alternative associated with delayed primary reinforcement.

Parallel findings exist in the human literature showing a link between income and temporal discounting. For instance, research has found that lower income adults discount delayed rewards more steeply than do higher income adults (Green, Myserson, Lichtman, Rosen, \& Fry, 1996). The present findings also correspond to findings with humans that people with higher needs (lower socio-economic status) tend to gamble proportionally more than those with lower needs (higher socio-economic status) (Lyk-Jensen, 2010).

Although, by itself, the conditioned reinforcement account provides a reasonable explanation for the preference for the low probability of reinforcement alternative, it fails to consider the conditioned inhibition that should accrue to the stimulus associated with the absence of reinforcement. Furthermore, in the case of the procedure used by Stagner and Zentall (2010), in which the conditioned reinforcer occurred on only $20 \%$ of the trials, conditioned inhibition 
should have been even greater, given that the signal for the absence of reinforcement occurred four times as often as the signal for reinforcement and, yet, the preference for the discriminative-stimulus alternative was almost $100 \%$. However, it appears that under the present conditions, conditioned inhibition is weak, relative to conditioned reinforcement, and is insufficient to overcome the higher value associated with the stimulus that predicts $100 \%$ reinforcement, relative to the alternative associated with $75 \%$ reinforcement. Interestingly, consistent with this conclusion, there is evidence that human gamblers attend to their wins much more than to their losses (Blanco, Ibáñez, Sáiz-Ruiz, Blanco-Jerez, \& Nunes, 2000). Furthermore, research on reward and punishment sensitivity in pathological gamblers has found that they have higher immediate reward sensitivity, as compared with controls (Franken \& Muris, 2005; Goudriaan, Oosterlaan, de Beurs, \& van den Brink, 2006; Petry, 2001).

The idea that an increase in motivation can have a negative effect on performance is reminiscent of the YerkesDodson law (Yerkes \& Dodson, 1908), in which it is proposed that there is a nonmonotonic relation (an inverted Ushaped function) between motivation and performance such that the optimal level of motivation for peak performance on a given task varies inversely with the difficulty of the task. According to this view, in the present experiment, one would conclude that the level of motivation of the pigeons in the high-restriction group was greater than optimal. However, to be consistent with the Yerkes-Dodson law, one would have to argue that the high-restriction group was less able to discriminate between $50 \%$ and $75 \%$ reinforcement. But if that were the problem for the high-restriction group, one would expect them to be indifferent between the two alternatives. Instead, they appeared to be more attracted to the immediate presentation of the stimulus that predicted $100 \%$ reinforcement than was the low-restriction group. Thus, one could view the task as involving two components: first, the establishment of the conditioned reinforcement (which appeared to be favored by the highrestriction group) and, second, the assessment of the overall probability of reinforcement given choice of the alternative (which appeared to be favored by the low-restriction group). If the optimal level of motivation for establishment of the conditioned reinforcer was relatively high and the optimal level of motivation for the assessment of the overall probability of reinforcement was relatively low, the Yerkes-Dodson law could account for the results of the present experiment.

Both the biopsychosocial model (Sharpe, 2002) and the pathways model of pathological gambling identify a group of gamblers who are behaviorally conditioned to gamble (Blaszczynski \& Nower, 2002), resulting in habitual patterns of gambling. These models further suggest that urges to gamble are acquired over time, further reducing the capacity for behavioral self-control, resulting in behavioral persistence despite negative consequences (Blaszczynski \& Nower, 2002; Sharpe, 2002).

The results of the present study (presented in Fig. 2) suggest that although pigeons in the low-restriction group generally preferred the optimal alternative once they had learned to associate the terminal stimuli with their outcomes, the preference declined with extended discrimination training, from a high of about $85 \%$ to a low of about $60 \%$. Although it is possible that if the experiment had been carried out longer, the pigeons in this group would have reached a stable level of indifference between the two alternatives, it is also possible that the preference would have ultimately shifted to the suboptimal alternative. That is, low levels of food restriction may have only limited ability to delay acquisition of a preference for the suboptimal alternative. In fact, recent unpublished research in our lab examining the effect of exposing pigeons to a socially enriched environment suggests that social enrichment results in the temporary choice of the optimal alternative but that, with additional experience with the task, pigeons acquire a preference for the suboptimal alternative. If pigeons in the lowrestriction condition come to exclusively choose the suboptimal alternative with extended discrimination training, this would call into question the Yerkes-Dodson law interpretation of these results and lend support for the pathways and biopsychosocial models of pathological gambling suggesting that urges to gamble are acquired over time. As the pathways model of human gambling would predict, this finding would yield support for the idea that problem gambling can emerge in the absence of impulsivity, since one can lose control over gambling in response to the effects of conditioning. Interestingly, the finding that pigeons in the low-restriction group began to lose their preference for the optimal alternative with continued exposure to the task suggests that it may be difficult for humans, even those who are not attracted to suboptimal risky choices, to resist gambling if they are exposed to gambling environments for an extended time. Thus, the similarity in results between pigeons with this suboptimal choice task and humans' gambling behavior suggests that both may have basic behavioral origins.

Author notes This research was supported by National Institute of Mental Health Grant 63726 and by National Institute of Child Health and Development Grant 60996. Correspondence should be addressed to Thomas R. Zentall, Department of Psychology, University of Kentucky, Lexington, KY 40506-0044. Email, zentall@uky.edu.

\section{References}

Ainslie, G. (1974). Impulse control in pigeons. Journal of the Experimental Analysis of Behavior, 21, 485-489. 
Alessi, S. M., \& Petry, N. M. (2003). Pathological gambling severity is associated with impulsivity in a delay discounting procedure. Behavioral Processes, 64, 345-354.

Belke, T. W., \& Spetch, M. L. (1994). Choice between reliable and unreliable reinforcement alternatives revisited: Preference for unreliable reinforcement. Journal of the Experimental Analysis of Behavior, 62, 353-366.

Blanco, C., Ibáñez, Sáiz-Ruiz, A., Blanco-Jerez, J., \& Nunes, E. V. (2000). Epistemology, pathophysiology, and treatment of pathological gambling. CNS Drugs, 13, 397-407.

Blaszczynski, A., \& Nower, L. (2002). A pathways model of problem and pathological gambling. Addiction, 97, 487-499.

Bradshaw, C. M., \& Szabadi, E. (1992). Choice between delayed reinforcers in a discrete-trials schedule: The effect of deprivation. Quarterly Journal of Experimental Psychology, 44, 1-16.

Eisenberger, R., Masterson, F. A., \& Lowman, K. (1982). Effects of previous delay of reward, generalized effort, and deprivation on impulsiveness. Learning and Motivation, 13, 378-389.

Franken, I. H. A., \& Muris, P. (2005). Individual differences in decisionmaking. Personality and Individual Differences, 39, 991-998.

Gipson, C. D., Alessandri, J. D., Miller, H. C., \& Zentall, T. R. (2009). Preference for $50 \%$ reinforcement over $75 \%$ reinforcement by pigeons. Learning \& Behavior, 37, 289-298.

Goudriaan, E., Oosterlaan, J., de Beurs, E., \& van den Brink, W. (2006). Psychophysiological determinants and concomitants of deficient decision making in pathological gamblers. Drug and Alcohol Dependence, 84, 231-239.

Green, L., Myerson, J., Lichtman, D., Rosen, S., \& Fry, A. (1996). Temporal discounting in choice between delayed rewards: The role of age and income. Psychology of Aging, 11, 79-84.

Herrnstein, R. J. (1981). Self-control as response strength. In C. M. Bradshaw, E. Szabadi, \& C. F. Lowe (Eds.), Quantification of steady-state operant behaviour (pp. 3-20). Amsterdam: Elsevier North-Holland.

Hull, C. L. (1943). Principles of behavior. New York: Appleton.

Kacelnik, A., \& Bateson, M. (1996). Risky theories: The effects of variance on foraging decisions. American Zoologist, 36, 402-434.

Krebs, J. R., \& Kacelnik, A. (1984). Time horizons of foraging animals. Annals of the New York Academy of Sciences, 423, 278-291.

Logue, A. W., Chavarro, A., Rachlin, H., \& Reeder, R. W. (1988). Impulsiveness in pigeons living in the experimental chamber. Animal Learning \& Behavior, 16, 31-39.
Logue, A. W., \& Peña-Correal, T. E. (1985). The effect of food deprivation on self-control. Behavioral Processes, 10, 355-368.

Lyk-Jensen, S. V. (2010). New evidence from the grey area: Danish results for at-risk gambling. Journal of Gambling Studies, 26, 455-467.

Molet, M., Miller, H. C., Laude, J. R., Kirk, C., Manning, B., \& Zentall, T. R. (in press). Decision making by humans in a behavioral task: Do humans, like pigeons, show suboptimal choice? Learning and Behavior. doi:10.3758/s13420-012-0065-7

Petry, N. M. (2001). Pathological gamblers, with and without substance use disorders, discount delayed rewards at high rates. Journal of Abnormal Psychology, 110, 482-487.

Petry, N. M., \& Casarella, T. (1999). Excessive discounting of delayed rewards in substance abusers with gambling problems. Drug Alcohol Dependence, 56, 25-32.

Rachlin, H., Logue, A. W., Gibbon, J., \& Frankel, M. (1986). Cognition and behavior in studies of choice. Psychological Review, 93, $33-45$.

Rachlin, H., Raineri, A., \& Cross, D. (1991). Subjective probability of delay. Journal of Experimental Analysis of Behavior, 55, 233244.

Sharpe, L. (2002). A reformulated cognitive-behavioral model of problem gambling: A biopsychosocial perspective. Clinical Psychology Review, 22, 1-25.

Snyderman, M. (1983). Optimal prey selection: The effects of food deprivation. Behavior Analysis Letters, 3, 359-369.

Stagner, J. P., \& Zentall, T. R. (2010). Suboptimal choice behavior by pigeons. Psychonomic Bulletin \& Review, 17, 412416.

Stephens, D. W. (1981). The logic of risk-sensitive foraging preferences. Animal Behaviour, 29, 628-629.

Stephens, D. W., \& Krebs, J. R. (1986). Foraging theory. Princeton, NJ: Princeton University Press.

Thorndike, E. L. (1933). A proof of the law of effect. Science, 77, 173 175.

Yerkes, R. M., \& Dodson, J. D. (1908). The relation of strength of stimulus to rapidity of habit- formation. Journal of Comparative Neurology and Psychology, 18, 459-482.

Zentall, T. R., \& Stagner, J. P. (2011). Maladaptive choice behaviour by pigeons: An animal analog and possible mechanism for gambling (suboptimal human decision making behaviour). Proceedings of the Royal Society B, 278, 1203-1208. 